\title{
Maestría en EduCACión: LOS IMPACTOS DE LA FORMACIÓN DE LOS PROFESORES DE LA REGIÓN DE la Chiquitania-Bolivia
}

\author{
MASTERS IN EDUCATION: THE IMPACTS OF TRAINING IN THE \\ TEACHERS IN THE CHIQUitaNia REgION - BOLIVIA
}

Mestrado EM EdUCAÇÁO: OS IMPACTOS DA FORMAÇÃo DE
PROFESSORES NA REGIÃo DA CHIQUITANIA - BOLÍVIA

Marialva Moog Pinto
Doutora em Educação. Universidade da Regiấo de Joinville - Univille. Brasil. ORCID: http://orcid.org/0000-0002-9898-8576 marialvamoog@hotmail.com

Fernando Rosas Montaño

Diretor do Programa de Pós graduación en Educación en la Universidad Latinoamericana - ULAT Universidad Latinoamericana - ULAT. Bolívia. ORCID: http://orcid.org/0000-0003-4922-418X

fer.rosas.edu@hotmail.com

Resumen: El presente estudio tiene como contexto el Curso de Maestría en Educación, hecho para profesores de la región rural de la Chiquitania, en Bolivia. El objetivo principal és analizar los posibles impactos a partir de la formación continua de los profesores/ estudiantes del Máster en esta región. El Curso proporciona la formación de gestores y profesores que actuan en los diversos niveles educativos de la región. Es una investigación cualitativa de naturaleza interpretativa que toma el contexto de la Chiquitania como el lugar de la investigación. El instrumento utilizado fue el cuestionario de preguntas abiertas y el análisis de los datos recopilados fue el análisis de contenido. El análisis mostró los impactos que esta formación tuvo en las concepciones de los estudiantes involucrados. Los datos analizados se presentan en categorías de análisis, organizados de la siguiente manera: en el campo personal; en el campo profesional y otros aspectos.

Palabras clave: Bolivia. Formación Contínua; Maestría en Educación; Región de la Chiquitania.

Aвstract: The present study has as context, the Master Course in Education, made for teachers of rural Chiquitania, Bolivia. The main objective is to analyze the possible impacts from the continuous training of the teachers / students of the Master in this region. The course provides the training of managers and teachers of education, professionals in different stages of education in the region. It is a qualitative research of 
interpretative nature that takes the context of Chiquitania as the locus of the research. The instrument used was the open questions questionnaire and the analysis of the collected data was the content analysis. The analysis showed the impacts that this formation had on the conceptions of the students involved. The analyzed data are presented in categories of analysis, organized as follows: in the personal field; in the professional field and other aspects.

KeYwords: Bolívia; Continuing Education; Masters in Education; Chiquitania Region.

Resumo: O presente estudo tem como contexto o Curso de Mestrado em Educaçáo realizado para professores da região rural da Chiquitania, na Bolívia. O objetivo principal é analisar os possíveis impactos da formação continuada de professores/alunos do Mestrado dessa regiáo. O Curso propicia a formação de gestores e professores da educação, profissionais em diferentes etapas de ensino, na região. Trata-se de uma pesquisa qualitativa de cunho interpretativo que toma o contexto da Chiquitania como o locus da pesquisa. $\mathrm{O}$ instrumento utilizado foi o questionário de questốes abertas e a análise dos dados coletados, foi a análise de conteúdo. A análise evidenciou os impactos que essa formação provocou nas concepçóes dos alunos envolvidos. Os dados analisados apresentam-se em categorias de análise, organizadas da seguinte maneira: no campo pessoal; no campo profissional e outros aspectos.

Palavras-Chave: Bolívia; Formação Continuada; Mestrado em Educação; Região da Chiquitania.

\section{Introducción}

La formacion continua responde a la necesidad de que los profesionales profundicen la formación y el conocimiento adquirido en la universidad o en los institutos de formación sean 'actualizados' de manera permanente para enfrentar los desafíos de un mundo competitivo. Es en este sentido que la universidad de la actualidad en general tiene una gama de cursos de Diplomado, Especialización, Maestría, Doctorado y Postdoctorado con la pretención de seguir mejorando las competencias profesionales para generar nuevas ideas, nuevos conocimientos, y sobre todo ser capaces de integrar los conocimientos en un mundo globalizado y exigente.

En el aspecto educativo el desafío de la innovación en el manejo del conocimiento, el uso de diversas fuentes de conocimiento, apropiación de las nuevas tecnologías aplicadas a la educación, son algunos de los motivos por los que se hace necesaria la educación continua. Esta realidad afecta a los profesionales y especialmente educadores de todo el mundo por lo que la formación continúa esta casi en todas las leyes y normas educativas de la edu- 
cación de los paises. Por una parte, el programa de formación sigue siendo importante porque posibilita que los maestros de esta región puedan acceder a un espacio de formacion para actualizar sus conocimientos, lograr competencias que les permita enfrentar los desafíos que la educacion demanda en el siglo XXI. Que la postmodernidad en el pensamiento de la población mundial no se extiende a estas regiones y a los maestros para contar con las herramientas necesarias para lograr una mejor educación para la población estudiantil de esta región. Por otra parte, el Programa de la Universidad Latinoamericana de Cochabamba cumple con una de las demandas más importantes sobre internacionalización de la educación superior, que es la movilidad de docentes y la divulgación del conocimiento para todos, pues trae docentes internacionales para contribuir en la formación.

Siendo asi, el Curso de formación, la Maestría en Educación, llega a los sitios lejanos, creando oportunidades para quien no tiene esperanza. Los estudiantes beneficiados son maestros con grado de licenciatura y algunos profesionales que se desempeñan en el área educativa en la región de la Chiquitania. El presente estudio tiene como objetivo principal analizar los posibles impactos en las concepciones de los estudiantes/profesores, logrados en el Curso de Maestría desde la formación en la comunidad de la Chiquitania en Bolivia.

Las preguntas de investigación se centran en saber si: ¿̨ienen los estudiantes en esta región otra forma de buscar la formación ofrecida por la Universidad Latinoamericana de Cochabamba? ¿Las reflexiones provocadas en el Curso cambian las concepciones de los estudiantes? ¿Los estudiantes se sienten desafiados a seguir en su formación?

A partir de las cuestiones anteriores, presentamos el problema de investigación que desea saber: ¿Cuál es el impacto del Curso de Maestría en la formación de los estudiantes/profesores de la comunidad de las Chiquitanías en Bolivia? En las preguntas de la investigación, es posible responder a las preguntas del estudio que muestra que los beneficios del programa son muchos, pero el principal es que, en el contexto de la realidad boliviana, el programa llena la ausencia de espacios de formacion en estas regiones y con ello satisface esta gran necesidad de formación continua en maestros que desempeñan su labor de manera muy sacrificada y que tienen una alta motivación para mejorar sus competencias profesionales.

A continuación, el estudio presenta los procedimientos científicos para llevar a cabo la investigación. 


\section{Metodología}

Este estudio es una investigación cualitativa de cuño interpretativo que toma como sujetos los entudiantes del curso de Maestría en Educación de la Universidad Latinoamericana que tiene su sede principal en Cochabamba-Bolivia, y excepcionalmente, con clases especiales, en las comunidades rurales de la región de la Chiquitania.

El curso se realiza en las ciudades de San José de Chiquitos con 30.120 habitantes, en Concepción con 18.800 habitantes, en San Antonio con una población de 6.481 personas y em Roboré que tiene 15.641 habitantes, todos en la región rural de Santa Cruz de La Sierra en Bolivia. La clase de maestría en San José atiende a 21 estudiantes, ya en Concepción la clase está compuesta por 48 estudiantes, en San Antonio los estudiantes son 20, y en Roboré, allí más cerca de la frontera con Mato Grosso en Brasil, hay 29 estudiantes. Asi la población de la investigación son 118 estudiantes y todos recibieron los cuestionarios por correo electrónico. Todos los estudiantes de la región rural del Curso, actúan de alguna manera en la red educativa, en diferentes niveles: primario, secundario, superior, además de gestores educativos y municipales. Los instrumentos utilizados para la recolección de datos, fueron cuestionarios de preguntas abiertas, ya que la muestra aleatoria fue de 25 sujetos.

El análisis de los contenidos proporcionados por los sujetos nos ayudó a entender los impactos que esta formación provocó en las concepciones profesionales, de los sujetos involucrados.

A continuación, el estudio trae al lector un entendimiento de la localidad en que ocurre la formación, como: El país Bolivia en el ámbito geopolítico y algunas de sus características regionales y educativas, asi como de la Región Rural de la Chiquitania.

\section{Contexto geopolitico de Bolivia}

Bolivia es un país con $1.098 .581 \mathrm{~km}^{2}$ de superficie, cuya variedad geográfica está nitidamente dividida en 3 zonas: la zona de los Andes, con los departamentos de La Paz, Oruro y Potosí; la zona central o de los valles, con los departamentos de Cochabamba, Chuquisaca y Tarija; y la región 
de los llanos orientales conformada por los departamentos de Pando, Beni y Santa Cruz. Esta variedad geográfica no ha permitido históricamente que el Estado y las universidades lleguen con educación a los lugares más alejados de esta amplia geografía boliviana, principalmente a la zona de los Llanos. Es por esta razón que en esta región, que ademas es geograficamente la más extensa pero menos atendida desde el pundo de vista educativo.

Sin embargo, debido a las largas distancias y la difícil vinculación de las comunicaciones, los profesores de la región no tienen posibilidades de acceder a las universidades para su formación continua, ya que las mismas solamente se encuentran en la capital de departamento: Santa Cruz de la Sierra.

Santa Cruz de la Sierra es el segundo departamento con el mayor número de profesionales de enseñanza con 39.678 profesores, después de La Paz, que tiene 51.558 profesores, y la tercera es Cochabamba con 31.345 profesores, según información del Instituto Nacional de Estadísdica (INE, 2017), como está demonstrado en el Gráfico 1.

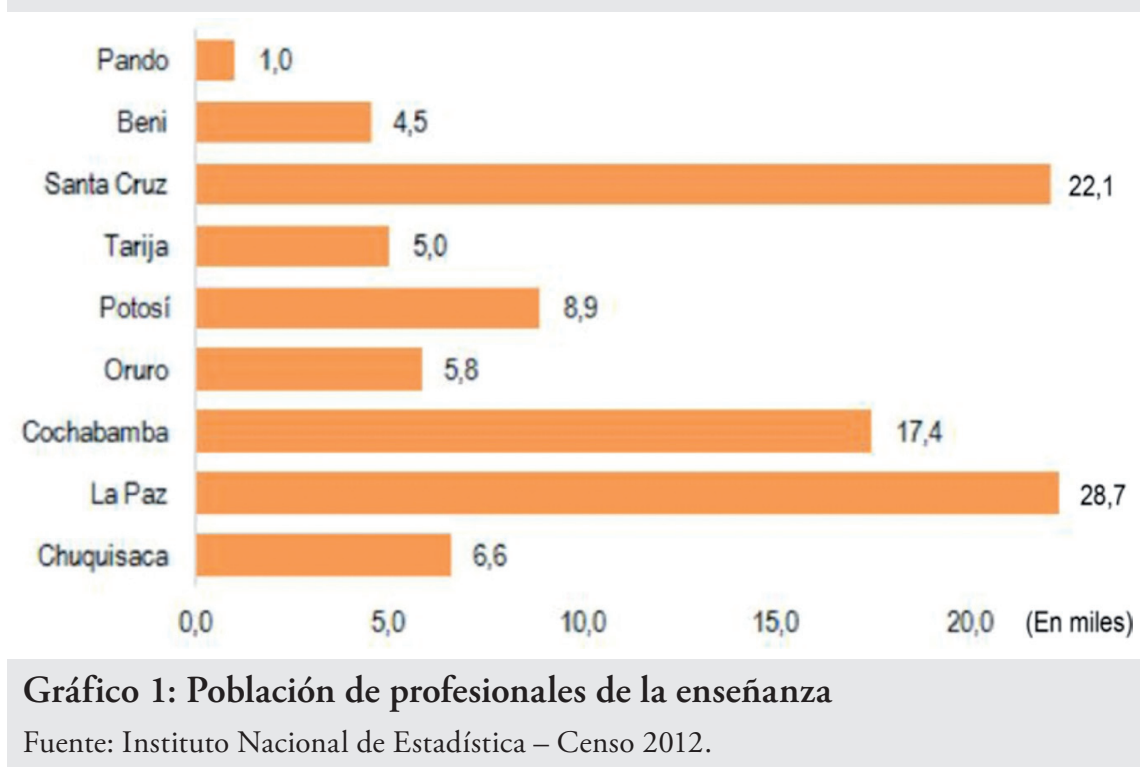

Esta realidad socioeducativa presentada en el gráfico anterior ha motivado a la Universidad Latinoamericana a ofrecer a los maestros de es- 
tas regiones y particularmente de las Chiquitania que conducen a maestria en la modalidad semi-presencial.

\section{La Región de la Chiquitania}

La Chiquitania boliviana es una vasta región ubicada al noreste del Departamento de Santa Cruz de la Sierra. La historia que contextualiza este lugar comienza a fines del siglo XVII con la llegada de los Jesuitas, quienes crearon las misiones con la finalidad de evangelizar a los nativos del lugar y asi consolidar la religión Católica en esta región. Entre 1691 y 1760 en la provincia de Chiquitos se construyeron diez misiones indígenas (Klein, 2015), como en la Figura 1. La región en este momento tenía aproximadamente 20.000 indígenas y allí construyeron unas magníficas misiones e iglesias que sobrevivieron hasta la fecha (Klein, 2015).

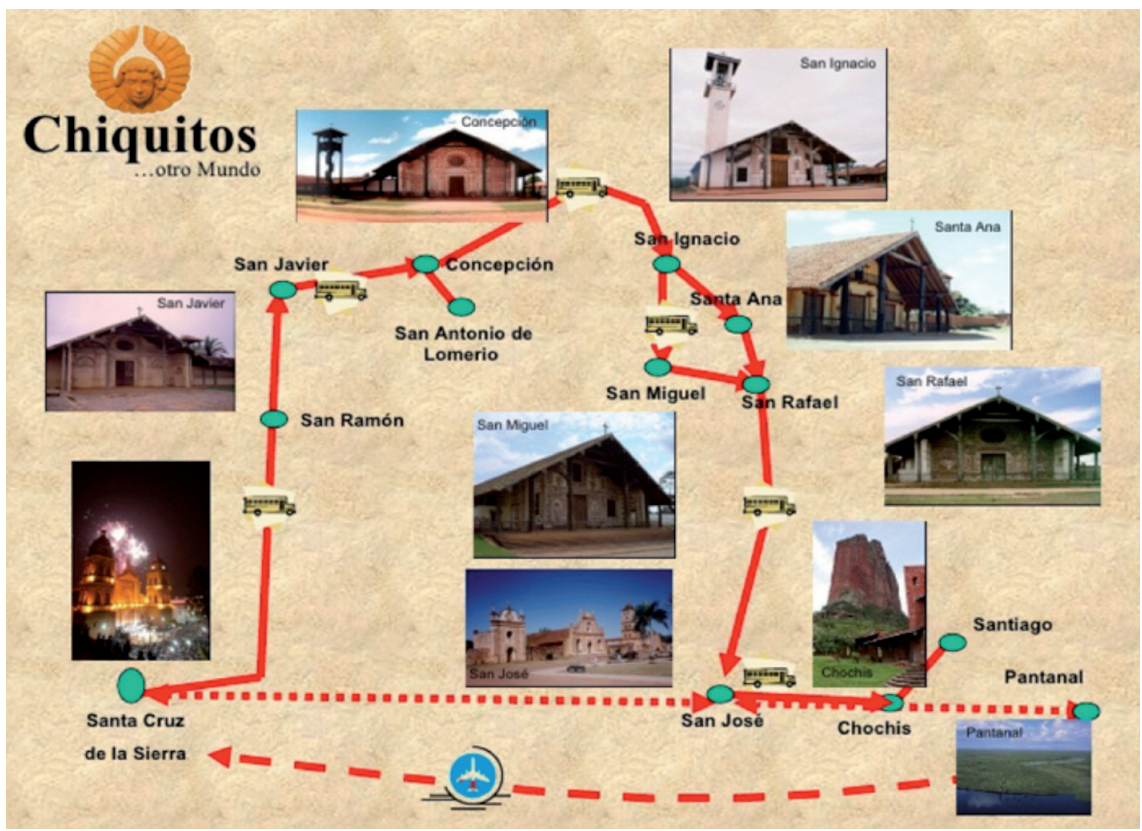

Figura 1: Las misiones de la Chiquitania

Fuente: Micha, 2009. 
Es sabido que el descubrimiento de América comenzó una etapa de exploración y colonización por espańoles y portugueses; las misiones tuvieron una singularidad en sus acciones, ya que San Ignacio de Loyola, fundador de la Compañía de Jesús, logró propósitos fundamentales en um nuevo mundo.

La Compañía de Jesús tuvo como uno de sus objetivos primarios, ejercer la defensa de la Iglesia Católica, especialmente ante el surgimiento en Europa de la Reforma Luterana y se convirtió en uno de los principales impulsores de la llamada Contrarreforma. (ESCUELA DIGITAL, 2014, p.1)

Estas misiones inicialmente lograron sembrar a los pueblos indígenas, ya que estos tenían una vocación nomada y el comienzo de una evangelización que desestructura la forma de vida y las creencias de los nativos. La magnitud de este abarcó una gran región geográfica.

La Compañía de Jesús, [...] formó una clase de misioneros tan especial, que pronto se destacó entre todas las órdenes. La rigurosa preparación y disciplina de sus miembros, el orden jerárquico existente entre ellos y los profundos estudios a que se dedicaban, tanto en las ciencias teológicas como en las ciencias exactas y naturales, prepararon a un conjunto de hombres que en pocos ańos se destacaron en los territorios donde desarrollaban su misión: Asia, África y América. En el Nuevo Continente extendieron su acción desde Canadá y Alaska hasta el Brasil y la Patagonia. (PELLINI, 2017, p.23)

En América del Sur, abarcaron principalmente parte de los territorios de Bolivia, Argentina, Brasil, Uruguay y Paraguay.

Los padres jesuitas llegaron a establecer muchas otras Misiones sobre las costas orientales del Río Uruguay, abarcando territorio de los actuales Departamentos de Artigas y Rivera, como del sur del actual estado brasileño de Río Grande del Sur; entre ellas las poblaciones de San Borja, San Ángel, San Juan, San 
Nicolás, San Luis, San Lorenzo y San Miguel, que alcanzaron en su conjunto una población superior a las 30.000 personas. (ESCUELA DIGITAL, 2014, p.1)

En la Figura 2 se puede mirar el mapa de las misiones jesuitas en América del Sur.

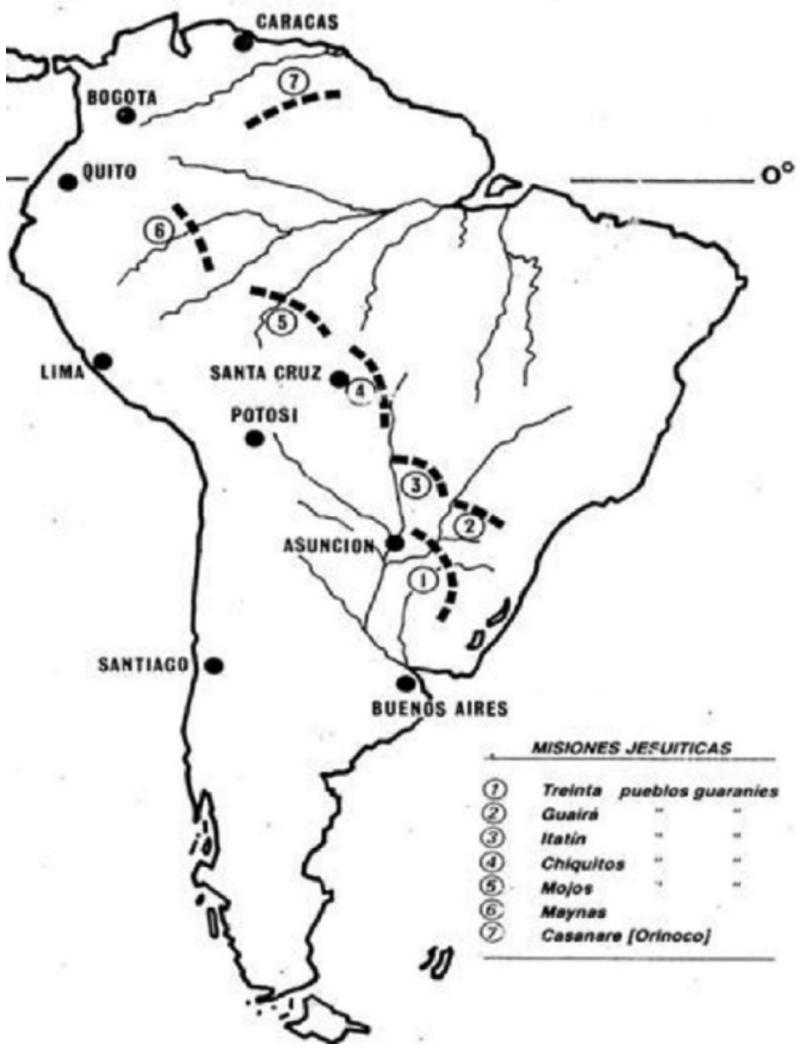

Figura 2: Misiones jesuitas en América del Sur Fuente: (Misiones, 2012, p. 1).

En el aspecto educativo durante la colonia, la región de las misiones de la Chiquitania fue un escenario de contradicciones. Por un lado, la autoridad de la iglesia representada por los jesuitas buscó la evangelización 
de los habitantes y por el otro estaban los indígenas enraizados con sus creencias y practicas heredadas de su cultura y sus antepasados, por lo tanto, este choque cultural fue desastroso, por lo menos, desde la perspectiva de una mirada crítica del tema.

La Iglesia Católica participó en la colonización de América, poniéndose al servicio de la política del régimen colonial. Estas son contradicciones en el enseńanza de que no pudo borrar del todo la ritualidad ancestral de los pueblos originarios. Los pueblos indígenas eran misiones religiosas bastante incivilizadas en la tierra de la doctrina cristiana, que les enseñaban artesanías españolas y formas de organización social. Todo eso se practicaba en las reducciones de tierras bajas, donde los grupos originarios eran sometidos a severas normas establecidas por los misioneros para el 'buen comportamiento' de los indios cristianizados. La reducción abarcaba la educación y la producción económica. (CHOQUE, 2013)

Em este contexto de los antecedentes de la Chiquitania, en la actualidad ha habido pocos cambios desde entonces, y la perspectiva de una educación con mejores opciones para el desarrollo estan todavia, sin embargo, en una etapa de construccion a paso muy lento.

\section{Programa Cooperación Belga en Bolivia mediante la Asociación para la Educación y la Formación en el Extrangero (APEFE)}

El esfuerzo educativo del Estado boliviano en la Chiquitania es todavia insuficiente, especialmente en lo que se refiere a la educación superior, pues se limita a la existencia de algunos institutos de educación técnica en Concepción y San José, por lo que la enseñanza universitaria es inexistente.

Esta realidad ha motivado a diferentes instituciones y principalmente ONGs a motivarse a ejecutar diferentes programas de capacitación a la zona. Es asi que el año 2013, la Cooperación Belga en Bolivia mediante la Asociación para la Educación y la Formación en el Extrangero (APEFE), ve la necesidad de mejorar las competências y elevar el nivel académico de los profesores. Estos maestros que se desempeñan en contextos rurales de "tierras bajas" eran solamente egresados de las Escuelas Normales y no 
habian obtenido aun la Licenciatura; las posibilidades de que esto ocurra eran una tarea imposible, ya que las universidades, como dijimos, estan en la ciudad capital, Santa Cruz de la Sierra. En esta dinâmica, luego de la firma de convênios de cooperacion específicos entre el gobiernos de Bélgica y Bolivia, la APEFE en alianza con la Universidad Pedagógica de Sucre organiza el Programa de Licenciatura para maestros bajo la supervicion del Ministerio de Educación como esta demonstrado en la Figura 3.

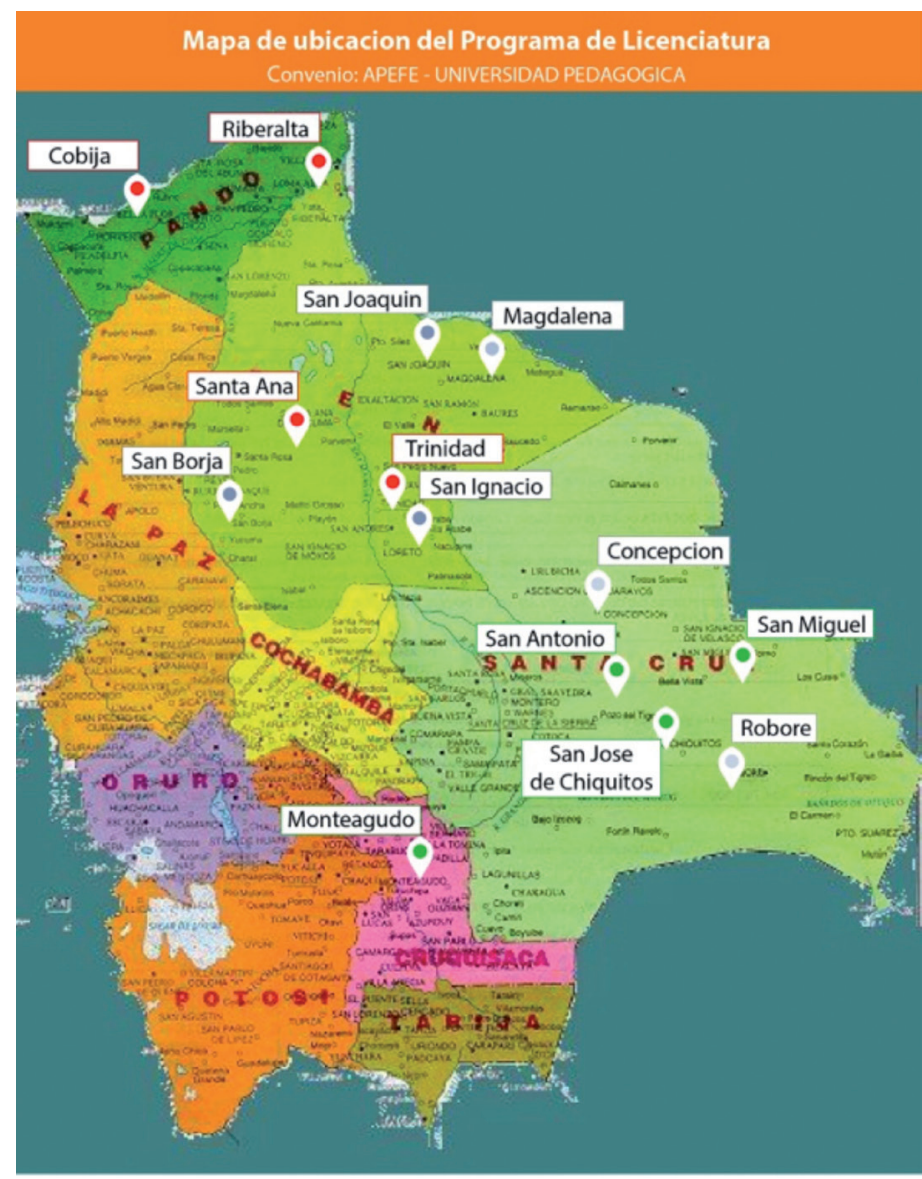

Figura 3: Lugares del Programa licenciatura

Fuente: Fuente primaria - Los autores. 
El programa fue llevado adelante con un exito sin precedentes en todos los aspectos y más de 600 maestros lograron su licenciatura. Este emprendimiento fue imitado por el Ministerio de Educación ya que la gran mayoría de los profesores de las áreas urbanas y rurales del sistema público normalistas no tenian la licenciatura. Para esta acción se crea el Programa de Formación Complementaria para Maestros para Licenciatura $\left(\mathrm{PROFOCOM}^{2}\right)$ la misma que se va desarrollando en casi todo el territorio nacional.

Sin embargo, la formación continua y de post grado, sigue siendo una necesidad para estos maestros y es aqui que la Universidad Latinoamericana en Cochabamba, recoje la demanda de profesores de la región de la Chiquitania que ya fueron formados en licenciatura y buscan para optar por cursos de diplomado conducentes a la maestria en educacion.

Esta región, de un pasado de permanente lucha contra los diferentes sistemas de dominación, tiene en sus profesores a personas motivadas en la búsqueda del desarrollo de habilidades y competencias para enfrentar mejor a los desafíos de la posmodernidad.

[...] la educación ahora más que nunca tiene un papel importante que jugar para que transformemos nuestro modo de pensar y de actuar y podamos afrontar la complejidad progresiva, la vertiginosidad de los cambios culturales, políticos, sociales, económicos, tecnológicos y comunicacionales que se van produciendo actualmente a nivel mundial. (GARCIA, 2001)

Actualmente maestros de San Antonio, San José de Chiquitos, Concepción y Roboré van a caminar para seguir superando para mejorar su desempeño profesional.

Tendo el leitor, entendido el contexto y la situación geopolítica en que la investigación ocorre, presentando a seguir, las análisis realizadas a partir de los datos recopilados en los cuestionarios echos a los estudiantes, para saber los posibles desafíos provocados por el Curso de Maestría a partir de la formación de los profesores de la comunidad de la Chiquitanía en Bolivia. Asi que los achados estan organizados en categorias de los aspectos personales y los aspectos profesionales. 


\section{Impactos en las concepciones de los profesores por la formación continua}

$\mathrm{Al}$ analizar las palabras de los sujetos cuestionados sobre los aspectos que el Programa de Maestría está influyendo y/o influenció en sus vidas personales y profesionales, fue posible encontrar algunas categorías de análisis, que son: en el ámbito Personal, en relación a sí mismo, y en relación con el contexto en el que se inserta; en el ámbito Profesional, como humanización, criticidad, la prácticas docentes, formación continua, profesor investigador, internacionalización; aspectos que deben plantearse como profesionales; y la expansión de la visión de futuro.

Por lo tanto, se presenta la continuación, las categorías analizadas:

\section{a) Aspecto personal}

En el Aspecto Personal, hay cambios en relación a sí mismo que llamaremos aspectos intrapersonales, y en relación con el contexto en que se insertan, que llamaremos aspectos interpersonales.

\section{Aspecto intrapersonal}

En este aspecto fue posible percibir que muchos estudiantes sienten un "cambio de actitud y personalidad" (P. Ana), han cambiado su forma de actuar, también han cambiado su forma de entendimiento sobre los hechos y sobre sí mismos. Dicen que hoy tienen "otra visión personal." (P. Julia)

Otro punto significativo para un estudiante fue el cambio en su vida financiera, porque a medida que expande las posibilidades intelectuales, mayor es su área de actuación. La estudiante (P. Ana) dice que el Programa de Maestría:

ha marcado de alguna manera los límites de mi realidad, por ejemplo : el factor económico, si en algún momiento es un obstáculo para continuar, pero también se vieron momientos en los que tuve que hacer un buen plan económico y pude subsanar este límite siendo que aquí tengo ventajas y desventajas también uno de las ventajas es que yo ya puedo ayudar 
mi economía haciendo trabajos extras pero bien relacionados con los estúdios, por ejemplo, puedo elaborar proyectos', dar talleres o asesorar trabajo, etc. y las desventajas ya van disminuyendo como por ejemplo no alejarme del contexto de estudios, investigar y leer más me ayuda a sentirme más capacitada.

Otro interlocutor investigado, también demuestra cuánto el Curso ha ampliado sus posibilidades personales pues hizo "desperta imaginación y puedo idear diferentes soluciones a los problemas y el desarrollo de ideas." (P. Pedro)

Según la Professora Priscilaima, "mi formación personal es una formación autocritica y analítica porque me permite crecer intelectualmente, es decir una alimentación para mi cérebro." (P. Priscila) Esto demuestra cuánto una formación intelectual a lo largo de la vida puede contribuir a la vida cotidiana de las personas, haciéndolas sujetos de sus propias vidas y a sus propias elecciones y no solo participando en las decisiones de los demás. (FREIRE, 1998)

\section{Relaciones interpersonales}

Los estudiantes relatan que el Curso há influido "en distintos ámbitos de la vida”, lo que nos muestra un cambio, pues los estudiantes han estado en una situación de reflexión, de intercambio en el grupo que se inserta y principalmente en un espacio de reflexión científica que les permite pensar más allá del sentido común. Otro, reafirma eso, diciendo que "influye en la forma de pensar hacia el futuro, porque es de manera reflexiva las clases que desarrollan.” (P. Priscila)

Las discusiones provocadas en el grupo, siempre han sido una propuesta política, social frente a la vida, al contexto y eso motiva a la responsabilidad por los problemas deste tiempo, como agentes responsables de contribuir en la resolución de los problemas contemporáneos. En el habla de una estudiante se percibe este compromiso cuando dice que "me siento más comprometida con la vida comunitaria, es decir, el Programa de Maestría ha creado en mi más empatía, motivación e implicación en todos los sentidos." (P. Ana) 


\section{b) Aspectos profesionales}

Los aspectos profesionales se han subdividido en otras categorías para ser analizados de forma detallada, ya que la riqueza de la información de los estudiantes no se puede simplificar.

\section{Humanizar y humanizarse}

En los relatos de los sujetos investigados, docentes/estudiantes del Máster, fue posible darse cuenta de la importancia que tiene para los mismos, la formación humana e integral. En este aspecto, se percibe que les importa formar a sus estudiantes como personas humanas, pero también para ellos, maestros, humanizarse. Este deseo, mencionado por uno de los estudiantes, viene al encuentro de las dos principales funciones de la Educación Superior: formar técnicamente, para ser un profesional competente; y humanizar a los sujetos. (Pinto, 2010)

Esta segunda función - humanizar a los estudiantes de la Educación Superior- es mencionada por los maestros/estudiantes cuando dicen que "en la tarea de ayudar a construir la mente de los estudiantes." (P. Ana) Y aún, "es muy importante en mi vida profesional porque me hace una persona más preparada para ayudar a niños y niñas en su formación integral.”

Otra estudiante dice que "el cambio de actitud y profesionalismo como persona, para servir a la sociedad y a la comunidad, siendo el ejemplo para la familia y especialmente a mis hijos, para que ellos sigán mis pasos y sean en el decir, mejores que yo." Otra estudiante todavía dice que "por otra parte estas clases nos permiten muñirnos de experiencias significativas para poder seguir caminando en este proceso de aprendizaje de la vida." (P. Paula)

La universidad "es conservadora, regeneradora y generadora de conocimiento, lo que estimula a hacer, en el presente, una articulación entre el pasado y el futuro, adoptando como práctica la crítica consciente, que es una consecuencia de la humanización del sujeto" (PINTO, 2009, p.172 apud MORIN, 2000). El autor dice además que la Educación Superior busca la humanización como sentido. La humanización es "la posibilidad de que todos los seres humanos tengan condiciones de ser partícipes y gozosos de los avances de la civilización históricamente construida y comprometida con la solución de los problemas que esa misma civilización generó." (PINTO, 2009, p.172 apud PIMENTA; ANASTASIOU, 2002) 


\section{Criticidad}

La criticidad es realmente un tema importante una vez que el tornar crítico, no es tarea fácil. Un estudiante dice: "me siento más capaz de enfrentar el tema de los debates enmarcados por la educación escolarizada y superior." (P. Ana). Según Freire (1998, p.34), "La superación y no la ruptura se da en la medida en que la curiosidad ingenua, sin dejar de ser curiosidad [...] critica, convirtiéndose entonces en curiosidad epistemológica [...] y connota sus hallazgos con mayor exactitud." Esta es una ganancia fundamental, que no siempre se puede medir, para el sujeto que busca una formación.

\section{Prácticas de enseñanza}

Se puede verificar en las respuestas de los interlocutores que ampliaron sus conocimientos, y los llevan a sus clases y para sus estudiantes "transmitiéndoles los temas de contenido concreto." (P. Ana) Otro sujeto investigado demostró haber cambiado su forma de entender el proceso de enseñanza y aprendizaje, pues ahora piensa en "enseña a aprender" ( $\mathrm{P}$. Ana). Este sujeto ya no ve el proceso como estático, sino como algo continuo que se puede aprender a lo largo de la vida.

Una de las maestras, que ocupa el cargo de Directora de una escuela, dice que:

Los planteamientos teóricos, metodológicos e instrumentales del programa de Maestría será um modelo de referencia para mi labor Directivo, en este contexto histórico de atención a la diversidad, de urgentes respuestas a un trabajo potenciador e incluso de los estudiantes con riesgo de exclusión o de fracaso escolar. (P. Ana)

Otro estudiante contribuye cuando dice que:

Esta influyendo de manera práctica por qué aplico en mi trabajo ya que trabajo en el área Educativo Administrativo. Por lo tanto actualmente estamos en el proceso de cambio, y a mi formación llega como anillo al dedo como dicen en esta zona, porque todo lo aprendido lo estamos aplicando. (P. Vivian) 
Esto también nos ayuda a compreender y creer siempre en el potencial de la formación continua en la cual el sujeto, a medida que construye nuevos conocimientos, los utiliza para revisar su práctica, innovar y repensar su saber hacer.

En las palabras de los estudiantes del Curso, está claro que se gana mucho de la formación, para cada uno en cada momiento algo produce um significado que a priori no podemos evaluar, pero se sabe que hay una práctica docente crítica a partir de eso.

Para la maestra, com respecto a la formación, dice que "cada módulo que nos imparten es de muchos beneficios, las experiencias adquiridas me permiten mejorar cada vez más el desarrollo de mis clases." Según Freire (1998, p. 42), "la práctica docente crítica, que implica un pensar correcto, involucra um movimiento dinâmico y dialéctico entre el hacer y el pensar sobre el hacer.”

Los avances también se producen en la forma en que los maestros planifican sus clases, en las metodologías que utilizan en clase, en la forma de evaluar, pues efectivamente el cambio ocurre en la concepción profesional de los maestros, de diferentes maneras y en tiempos distintos pero ocurren. Esto se confirma en el discurso de uno de los estudiantes que dice "como soy profesor de un Instituto de Educación Superior, todos los avances me han servido como base para optimizar mi metodología de enseñanza y más que todo en la parte de la escolaridad y los sistemas de evaluación." (P. Regina) El Curso de Maestría ayuda "en el aspecto educativo de la práctica diaria en el ámbito laboral" y "conocer los diferentes métodos de enseñanza y aprendizaje”, según el estudiante P. Pedro.

Según otro estudiante, sobre su vida diaria como gestor, dice que "así también he aprendido las herramientas para elaborar un Plan Operativo Anual (POA) y un Proyecto Educativo Institucional Comunitário (PEIC)" (P. Regina), que son documentos solicitados por las Secretarias de Educación a las escuelas.

\section{Formación continua}

La formación continua de profesores pasa por la condición en que ellos van asumiendo una identidad docente, lo que supone la asunción del hecho de ser sujetos de la formación, y no objetos de la misma, como meros instrumentos flexibles y manipulables en manos de los formadores. 
Segun un maestro, sobre la formación disse: "una de las experiencias más sobresalientes en mi vida profesional, ya que me sirve de apoyo en la acción docente." (P. Ana) Las transformaciones a partir de la formación profesional, pueden ser constatadas en las prácticas de estos maestros, en el sistema educativo en que actúan y aún en el contexto en el que se insertan. Todos se benefician de la formación continua de un profesional de la educación. Se puede confirmar lo dicho, a través de la habla de otro estudiante "que a través del estudio que nos brinda la maestría, es impartir y poner en práctica los conocimientos que tengo hacia mis estudiantes, porque capacitar es mejorar en el trabajo y que los estudiantes se benefician." (P. Julia)

La implicación de una formación continua está en muchos ámbitos de la práctica profesional porque "me ayuda a planificar, enseñar y tomar otras estrategias innovadoras para fortalecer el aprendizaje y la enseńanza." Según este estudiante y el otro, la "formación de habilidades para llevar a cabo el trabajo de planificación, desarrollo y evaluación.” En este sentido, el programa de Maestría está orientado a viabilizar la práctica pedagógica, de manera propicia en el arte de la enseñanza, según P. Luisa.

Este estudio, cuando habla sobre el ser humano que nunca deja de aprender, trae el concepto sobre el "aprendizaje a lo largo de la vida". El dice que su formación da como ejemplo para los estudiantes "que el ser humano nunca deja de aprender, cada momento adquiere el conocimiento sea de una manera empírica o científica." (P. Ana) Esto nos remite al documento europeo sobre la política de formación, el Memorandum sobre la educación y la formación a lo largo de la vida, ratificado en marzo de 2000, en Lisboa, por la Comisión de las Comunidades Europeas (2000, p.3), que define:

aprendizaje a lo largo de la vida (lifelong learning) no es sólo uno de los aspectos de la educación y el aprendizaje; Debe convertirse en el principio director que garantiza a todos el acceso a las ofertas de educación y formación en una gran variedad de contextos de aprendizaje.

La formación continua requiere un clima de colaboración y el maestro que acepta un proceso de formación continua, debe hacerlo porque cree que es importante. Debe ser pensado siempre con respecto a aquellos 
que poseen la experiencia local, contextual, con participación de todos los miembros y un liderazgo democrático. No se impone la formación para quien piensa que lo que hace ya va bien, pues la formación no anda paso a paso con la resistencia.

\section{Profesor investigador}

Las respuestas de muchos estudiantes muestran claramente la valorización que los mismos perciben sobre el proceso de investigación sobre sus prácticas. Uno de los estudiantes entiende que "con nuevos conocimientos en la enseñanza y aprendizaje de diferentes actividades también en la investigación" (P. Luisa); otro demuestra su interés en investigar pues eso ayuda a "mejorar la calidad educativa en el proceso de enseñanza y aprendizaje, tanto pedagógico como de valores." (P. Ana) También dicen que ser un profesor investigador les da otra visión personal de su profesión.

En la visión de Shön (2000), esta es la postura del profesor reflexivo, aquel que refleja sobre su acción docente no sólo durante el desarrollo del trabajo, sino también después de su término. En la concepción de Shulman (1986), el maestro se convierte en un verdadero profesional, cuando es capaz de comprender, reflexionar, adaptarse- y criticamente- programar sus acciones.

\section{Internacionalización de la Educación Superior}

Los estudiantes, al contar con la participación de una profesora de Brasil que estuvo ministrando clases en el Curso, comentan que el proceso de internacionalización "está influyendo en su status, al tener acceso a una amplia gama de información e interactuar con los docentes del internacionais." (P. Regina)

La movilidad de estudiantes y profesores en la educación superior y los programas de investigación, es uno de los criterios para una educación superior de calidad, según lo acordado en las últimas conferencias mundiales sobre educación superior, en organizaciones como a United Nations Educational, Scientific and Cultural Organization (UNESCO) y Organisation for Economic Co-operation and Development (OECD), que están involucradas.

Uno de los objetivos de esta propuesta es la libre divulgación y intercambio de conocimientos. Sin embargo, en Bolivia, por entendimiento 
político, este acuerdo no encuentra libre circulación y entendimiento, quedando a cargo de iniciativas individuales, como el caso del Programa de la APEFE en cooperación con la Universidad de Sucre y posteriormente con la Universidad Latinoamericana de Cochabamba, proporcionar esa movilidad.

Concluyendo el análisis, fue posible aún compreender que los estudiantes mencionaron aspectos a mejorar en su profesión y su visión de futuro después de esa formación.

\section{Aspectos personales y profesionales para mejorar}

Es posible percibir que los estudiantes a medida que se realizaba la formación, se dan cuenta de que algunos puntos deben mejorarse como profesionales. Tal vez esto se les haya dado desde su punto de vista grupal, pues ya no son sólo los niños que los observan, y en el caso de los compañeros maestros, las demandas personales se están volviendo más evidentes.

Una de las maestras dice "solo tengo que perder un poco más de timidez y tener más fluidez en hablar" (P. Ana) y el otro dice "esta influyendo en mi vida personal en el cambio de las actitudes, el comportamiento, y la forma analítica al emitir expresiones verbales y escritas." (P. Vivian; P. Pedro) Otra dice que necesita mejorar su autoestima.

Hay una satisfacción cuando relatan que están ampliando su vocabulario, con términos nuevos, términos técnicos, y sus significados.

\section{Visión del futuro}

La formación fomenta su visión del futuro, sus deseos de seguir calificándose:

Yo quiero ser una docente ejemplar demostrando mi capacidad intelectual con valores éticos profesional, no solamente en el aula sino también demostrando mi profesionalismo hacia la sociedad y la comunidad, porque el ser Maestro es el ejemplo del estudiantes y el encargado de la transformación de muchos estudiantes. (P. Ana)

$\mathrm{Y}$ es el deseo de estar "mejor preparada para competir con otros profesionales de diferentes rubros del país y con profesionales internacio- 
nales." (P. Ana) Otro estudiante se siente fortalecido para en el futuro "trabajar en otras instituiciones superiores" (P. Priscila), con su mirada hacia otros espacios profesionales.

Sin embargo, hay estudiantes que quieren mejorar para seguir en su propio contexto y mejorar aún más lo que está haciendo, y si puede percibir eso en el discurso de uno de los estudiantes que dice sentirse bien "de seguir preparándome en el manejo de la tecnología ya que los niños cada dia, estan más hábiles.” (P. Ana)

\section{Consideraciones finales}

Al realizar este estudio con el objetivo de saber cuáles son los posibles impactos en la formación de los estudiantes/profesores del Curso de Maestría fomentado por la Universidad Latinoamericana, no hay duda de que hubo muchos impactos.

El Curso ha ofrecido un cambio de concepción en los profesores que participan en la formación, pero antes de todo, el Curso ha provocado la praxis profesional. Los profesores/estudiantes del Curso, a medida que han pensado sobre sus prácticas, la modifican con una mirada más profesional.

Se percibe que además de los cambios profesionales la reflexión también ha provocado cambios personales e intentos de actuar en el contexto en el que se insertan positivamente, además de la posibilidad de seguir formándose.

Aunque muchos tienen dificultades financieras para seguir formandose, se percibe que la formación ha expandido su área de actuación profesional, ampliando también las posibilidades de ganancias.

El Curso produjo reflexiones epistemológicas y menos del sentido común, con una mayor visión y entendimiento para resolver problemas personales y de la vida en comunidad. Los estudiantes se sienten cargo por su localidad, pues saben la importancia de la formación y la imposibilidad que otros tantos tengan para estar en su conjunto, lo que los hace responsables y críticos de la realidad.

Los estudiantes/profesores son conscientes de que existe una mayor responsabilidad por la formación técnica de calidad y entienden que sus estudiantes son sujetos integrales. 
Se sienten más seguros profesionalmente, teniendo como modelo de formación el propio Curso de Maestría y sus docentes. Aplican todo lo aprendido para mejorar el desarrollo de sus clases, entendiendo mejor el proceso de enseñanza y aprendizaje, como se planea una clase y las formas de evaluación.

Otro punto importante a tener en cuenta es que los estudiantes han desvelado el contexto, ampliaron su visión del futuro, vislumbrando otros espacios formativos educativos nacionales e internacionales nunca antes pensados, lo que puede ser una ganancia para la sociedad local que no podemos medir a priori.

\section{Notas}

1 Asi tambien es denominada la region de los llanos orientales de Bolivia

2 El Programa de Formación Complementaria para Maestras y Maestros (PROFOCOM) ha sido el programa del Ministerio de Educación que ha asumido la tarea de, a través suyo, formar a las maestras y maestros del país en el nuevo currículo educativo del Estado Plurinacional.

\section{Referencias}

BOLIVIA. Ministerio de Educación: Estado Plurinacional de Bolívia. El PROFOCOM un Programa de Formación Complementaria para Concretizar la Revolución Educativa. Coleción Avances de La Revolución Educativa. La Paz. Bolívia. 2016.

BOLIVIA. Instituto Nacional de Estadística: Estado Plurinacional de Bolívia. Educación. Disponible en: http://www.ine.gob.bo/index.php/educacion/educacion-2 2017.

BOLIVIA. Instituto Nacional de Estadística: Estado Plurinacional de Bolívia. Censo de Población de Bolivia. Disponible en: http://censosbolivia.ine.gob.bo/webine/photogallery/bolivia-por-municipios-cnpv-2012-poblaci\%C3\%B3n . 2017.

CHOQUE, Roberto. La educacion en la colonia (vol. ii). La Paz: Instituto de Investigacion Plurinacional. La Paz. 2013.

PORTUGAL. Commission of the European Communities. (2000). A Memorandum on Lifelong Learning. Lisbon. Disponible en: http://context.reverso.net/traducao/ portuguesingles/sobre+a+Aprendizagem+ao+Longo+da+Vida 2017. 
ESCUELA DIGITAL. La Colonização del continente americano. Las Misiones Jesuitas. Disponible en: http://www.escueladigital.com.uy/historia/colonizacion/las_misiones. htm\#sel. s/p. 2017.

FREIRE, Paulo. Pedagogia da Autonomia: saberes necessários à prática educativa. 9 ed. São Paulo: Paz e Terra, 1998.

GARCIA, Dignora. Postmodernidad: desafios de la educacion. Disponible en: http:// www.centropoveda.org/img/pdf/postmodernidaddesafioseducacion.pdf 2017.

IMBERNÓN, Francisco. Formação continuada de professores. Porto Alegre: Artmed, 2010.

KLEIN, Herbert. S. Historia minima de Bolivia (vol. 1). Edición Kindle. Mexico: El Colegio de Mexico. 2015.

MICHA, Mariuxy. es.slideshare.net. Disponível en: https:/es.slideshare.net/patysama/ presentacin-informe-mariuxy-micha , 2017.

NAVARRO, Saturnino. satur-turismo.blogspot.com. Misiones Jesuiticas de América del Sur. Disponível en: http://satur-turismo.blogspot.com.br/2011/11/misiones-jesuiticasde-america-del-sur.html 2017.

PELLINI, Ana. Organización de las misiones Jesuiticas en America: Los Jesuitas. Disponível en: http://historiaybiografias.com 2017.

PINTO, Marialva Moog. Humanização e qualidade da educação superior: limites e possibilidades das políticas de inclusão. Revista Educação Unisinos. Ed. Unisinos. São Leopoldo. maio/agosto. p.169-175, 2009.

ROSAS, Fernando. Comision de pilotaje del programa de licenciatura. APEFE. Santa Cruz: APEFE. 2013.

SHÖN, Donald. Educando o profissional reflexivo: um novo design para o ensino e a aprendizagem. Porto Alegre: Artes Médicas, 2000.

SHULMAN, Lees. Those who understand: knowledge growth in teaching. Educational researcher tional researcher, v. 15, n. 2, p. 4-14. 1986.

Recebido em 24 fev. 2019 / Aprovado em I9 dez. 2019

Para referenciar este texto:

PINTO, M. M.; ROSAS MONTAÑO, F. Maestría en Educación: los impactos de la formación de los profesores de la región de la Chiquitania-Bolivia. EccoS-Revista Científica, São Paulo, n. 5I, eI2378, out./dez. 2019. Disponível em: https://doi. org/I0.5585/EccoS.n5I.I2378. 